\title{
Safety of Etanercept and Methotrexate in Patients with Rheumatoid Arthritis and Hepatitis C Virus Infection: A Multicenter Randomized Clinical Trial
}

\author{
Florenzo Iannone, Giovanni La Montagna, Gianfilippo Bagnato, Elisa Gremese, \\ AnnaRita Giardina, and Giovanni Lapadula
}

\begin{abstract}
Objective. To evaluate the safety and efficacy of therapy with etanercept and methotrexate (MTX) in patients with active rheumatoid arthritis (RA) and mild hepatitis $\mathrm{C}$ virus (HCV) infection.

Methods. In this prospective open study, 29 patients with active RA were randomly assigned to receive therapy with MTX alone, etanercept alone, or a combination of MTX and etanercept, and monitored up to 54 weeks. The primary endpoint was safety; secondary aims were efficacy as defined by the 44-joint Disease Activity Score (DAS44) and health assessment questionnaire (HAQ). Serum liver enzymes and HCV viral load were serially measured.

Results. In the whole cohort, aspartate aminotransferase (AST) serum levels were (mean \pm SD) 35 \pm 3 at entry, $39 \pm 5,41 \pm 7$, and $38 \pm 4$ at 14, 30, and 54 weeks, respectively; alanine aminotransferase (ALT) serum levels were $43 \pm 5$ at entry, $47 \pm 5,53 \pm 9$, and $50 \pm 6$ at 14, 30, and 54 weeks, respectively. $\mathrm{HCV}$ viral load was $5.6 \pm 0.5$ at entry, $5.9 \pm 0.6,5.7 \pm 0.3$, and $5.6 \pm 0.6$ at 14,30 , and 54 weeks, respectively. AST and ALT did not significantly change in all 3 arms of treatment, nor did HCV viral load. A significant reduction of DAS44 ( $<<0.01)$ and HAQ $(\mathrm{p}<0.04)$ was detected at 54 weeks compared to baseline. No patient discontinued the therapy because of worsening of liver disease.

Conclusion. This study showed that patients with RA and chronic HCV and mild hepatitis may be successfully treated with etanercept and MTX without increasing the risk of hepatotoxicity and HCV replication. ClinicalTrials .gov Identifier NCT01543594. (First Release Jan 15 2014; J Rheumatol 2014;41:286-92; doi:10.3899/jrheum.130658)
\end{abstract}

Key Indexing Terms:

TNF- $\alpha$ INHIBITORS

DISEASE ACTIVITY SCORE

TRANSAMINASES GISEA HEALTH ASSESSMENT QUESTIONNAIRE

A major concern in treating patients with rheumatoid arthritis (RA) is drug-induced immunosuppression, and when RA is associated with chronic infections, it becomes a therapeutic challenge for the rheumatologists. Hepatitis $\mathrm{C}$ virus (HCV) infection exists worldwide and occurrence in patients with RA is not uncommon, mainly in endemic areas. Upon infection with $\mathrm{HCV}$, patients with RA might

From the Interdisciplinary Department of Medicine, Rheumatology Unit, Bari; Rheumatology Unit, II University of Naples, Naples; Rheumatology Unit, Messina; Università Cattolica del Sacro Cuore, Rome;

Rheumatology Unit, University of Palermo, Italy.

Florenzo Iannone has received consulting fees, speaking fees or honoraria (less than $€ 10,000)$ from Pfizer, Merck, Abbott, Bristol-Myers Squibb, and $U C B$.

F. Iannone, $M D$, PhD, Associate Professor of Rheumatology, Interdisciplinary Department of Medicine, Rheumatology Unit, Bari; G. La Montagna, MD, Rheumatology Unit, II University of Naples, G. Bagnato, MD, Rheumatology Unit, Messina; E. Gremese, MD, Università Cattolica del Sacro Cuore; A.R. Giardina, MD, Rheumatology Unit, University of Palermo; G. Lapadula, MD, Interdisciplinary Department of Medicine, Rheumatology Unit, Bari.

Address correspondence to Dr. F. Iannone, Interdisciplinary Department of Medicine-Rheumatology Unit, Policlinico, Piazza G. Cesare 11, 70124 Bari, Italy.E-mail: florenzo.iannone@uniba.it

Accepted for publication October 1, 2013. experience both the worsening of liver function because of drug toxicity and an increase in HCV replication due to immunosuppression. This is particularly true for methotrexate (MTX), whose liver toxicity is well known and a possible cirrhotic effect requiring serial liver biopsies was documented some years $\mathrm{ago}^{1}$. However, to our knowledge, the combined prevalence of liver toxicity from MTX and liver damage from chronic HCV infection has not been reported to date. These concerns have increased since the use of tumor necrosis factor (TNF- $\alpha$ ) inhibitors for the treatment of RA, whose blocking effects may affect the competence of the immune system to control viral infection ${ }^{2}$. However, some reports have suggested that anti-TNF drugs are safe in patients with RA and concomitant HCV infection ${ }^{3,4,5,6,7,8,9}$. These studies were mainly case series and retrospective surveys and TNF blockers were given as monotherapy, except in the study by Bellisai, et al, in which anti-TNF drugs were combined with cyclosporine ${ }^{3}$. Randomized clinical trials are lacking regarding the safety and efficacy of combined therapy with anti-TNF drugs and MTX, the latter being the anchor drug for most biological drugs in the treatment of RA. In our

Personal non-commercial use only. The Journal of Rheumatology Copyright $($ C 2014. All rights reserved. 
multicenter randomized study, we evaluated the safety, mainly liver toxicity, and the clinical outcomes of therapy with MTX alone or etanercept alone, and the combined treatment MTX plus etanercept over 12 months.

\section{MATERIALS AND METHODS}

Patients. Patients fulfilling American College of Rheumatology (ACR) 1987 classification criteria for RA were prospectively enrolled in 5 rheumatology centers in southern Italy belonging to the Gruppo Italiano di Studio sulle Early Arthritides (GISEA). GISEA instituted a register in which information on patients with rheumatic diseases commencing biologic drugs is compiled (ClinicalTrials.gov Identifier NCT01543594). Approval was obtained by the local ethics committee for our study and the patients were recruited after giving their written informed consent in compliance with the Helsinki declaration.

Inclusion criteria were (1) age $\geq 18$ and $\leq 75$ years old, (2) MTX-naive patients with Disease Activity Score on 44 joints (DAS44) $\geq 3.7$, and (3) patients with $\mathrm{HCV}$ infection not requiring antiviral therapy. Exclusion criteria were (1) elevated serum transaminases $>3$-fold upper normal limits in the last 3 months, (2) liver cirrhosis diagnosed by liver biopsy or according to the clinical Child-Pugh score, (3) previous treatment with MTX or biological drugs, (4) presence of active or latent tuberculosis (assessed by chest radiographs and tine test), (5) active infections, (6) neoplasia, except for skin basocellular carcinoma, (7) classes III-IV of New York Heart Association heart failure, (8) pregnancy, and (9) concomitant $\mathrm{HBV}$ infection, including anti-HBcAg carriers.

$\mathrm{HCV}$ infection was diagnosed using commercial ELISA kits to detect anti-HCV antibodies and subsequent measurement of HCV viremia by polymerase chain reaction. Viral load was expressed as $\log 10$ of the detected values for analysis. The laboratory tests included measuring for rheumatoid factor, anticyclic citrullinated peptide, cryoglobulins, antinuclear antibody, antiextractable nuclear antigen, anti-smooth muscle antibodies, antimitochondrial autoantibodies, first-hour erythrocyte sedimentation rate, C-reactive protein, albumin, prothrombin time (PT), aspartate aminotransferase (AST), alanine aminotransferase (ALT), alkaline phosphatase (ALP), gamma-glutamyl transferase (GGT), and bilirubin.

The clinical data collected were age, sex, disease duration, smoking, number of swollen and tender joints, pain assessed by a visual analog scale (VAS 0-100), patient's assessment of general health (VAS, range 0-100), Ritchie's index, health assessment questionnaire (HAQ) scores, and side effects.

Design of the study. Our study was prospective and open-labeled, with a period of enrollment of 2 years. Eligible patients were randomly assigned to receive MTX alone (starting dose $10 \mathrm{mg} /$ week subcutaneously), etanercept alone (50 mg/week subcutaneously), or combined therapy with MTX $10 \mathrm{mg} /$ week and etanercept $50 \mathrm{mg} /$ week. The randomization was carried out according to an allocation concealment mechanism: 1 external person sequentially assigned by phone to the interventions $1: 1: 1$, while the allocation sequence was concealed to researchers. Concomitant treatments with glucocorticoids at doses of 5-7.5 mg/day (prednisolone or equivalent) and symptomatic nonsteroidal antiinflammatory drugs were allowed. For patients taking MTX alone or in combination who developed elevated serum transaminases or increase in $\mathrm{HCV}$ replication, rescue into the etanercept branch was permitted. At entry, 14, 30, and 54 weeks, serum liver function tests (AST, ALT, ALP, GGT, bilirubin, albumin, PT), and HCV viral load were measured, together with liver ultrasound (US) examination. DAS44 and functional disability (HAQ) were assessed at the same time. The European League Against Rheumatism (EULAR) clinical response criteria were used to evaluate the clinical response; accordingly, patients are divided into 3 categories: "good" response when the DAS44 change from baseline is $>1.2$, "moderate" when change is between $>0.6$ and $<1.2$, or "none" when change is $<0.6$.

Statistical analysis. A normal distribution of continuous variables was estimated by using the Kolmogorov-Smirnov test. Continuous variables were reported as means and SD if normally distributed, while medians and interquartile (IQR) range were calculated for non-normally distributed continuous variables. For categorical variables, counts and percentages were calculated. Differences in means for normally distributed continuous variables were compared by using ANOVA and least square difference posthoc analysis, or Student $t$ test for paired data. The Wilcoxon signed-rank test was used to compare non-normally distributed continuous variables. Differences in the distribution of frequencies were assessed by chi-squared test and Yates' correction where appropriate. Analyses were done using SPSS (version 20) statistical software; a $p<0.05$ was considered statistically significant.

\section{RESULTS}

Clinical data. Seventy-four patients with RA and HCV infection were screened and 38 of them were excluded because of elevated serum transaminases $>3$ times the upper normal limits or liver cirrhosis. Thirty-six patients with mild hepatitis were enrolled in the study, but 7 of them withdrew consent after the randomization, and 29 patients began the treatment. Three patients were obese (body mass index over $30 \mathrm{~kg} / \mathrm{m}^{2}$ ), but only 2 of them started the therapy. Of 4 patients with diabetes mellitus, 2 withdrew the consent, 1 dropped out of the study after 14 weeks, and 1 completed the study. Of 29 patients, 15 showed hepatomegaly and 12 steatosis on US examination of the liver. Clinical data and demographics are summarized in Table 1. At baseline, all patients were MTX-naive and had high disease activity with mean DAS44 > 3.7 and poor functional ability with a median HAQ $=1.25$. No difference in terms of disease activity, disease duration, serum HCV viral load, and liver enzymes among the 3 types of treatment was seen. Five patients were also taking low-dose prednisone $(5-7.5 \mathrm{mg}$ per day). The flow chart of the study is shown in Figure 1. Out of 29 patients, 9 began therapy with MTX alone, 13

Table 1. Clinical and demographic data of patients with rheumatoid arthritis (RA) at entry.

\begin{tabular}{|c|c|c|c|c|c|}
\hline & \multicolumn{5}{|c|}{ Patients with RA, $\mathrm{n}=29$} \\
\hline & $\mathrm{n}(\%)$ & Mean & $\pm 1 \mathrm{SD}$ & Median & IQR \\
\hline Age, yrs & & 55.4 & 13 & & \\
\hline Male & $8(27)$ & & & & \\
\hline Female & $21(73)$ & & & & \\
\hline Diabetes mellitus & $2(6.9)$ & & & & \\
\hline Obesity & $2(6.9)$ & & & & \\
\hline $\mathrm{RF}+$ & $25(86)$ & & & & \\
\hline Glucocorticoids & $5(17)$ & & & & \\
\hline Disease duration, yrs & & 6.2 & 2.4 & & \\
\hline VAS pain, $0-100$ & & 62 & 21 & & \\
\hline DAS44 & & 4.2 & 0.6 & & \\
\hline HAQ & & & & 1.25 & 0.75 \\
\hline $\mathrm{ESR}, \mathrm{mm} / \mathrm{h}$ & & 45 & 12 & & \\
\hline $\mathrm{CRP}, \mathrm{mg} / \mathrm{dl}$ & & 2.0 & 0.6 & & \\
\hline
\end{tabular}

RF: rheumatoid factor; ESR: erythrocyte sedimentation rate; CRP: C-reactive protein; VAS: visual analog scale; DAS44: 44-joint Disease Activity Score; HAQ: Health Assessment Questionnaire; IQR: interquartile range.

Personal non-commercial use only. The Journal of Rheumatology Copyright @ 2014 . All rights reserved. 


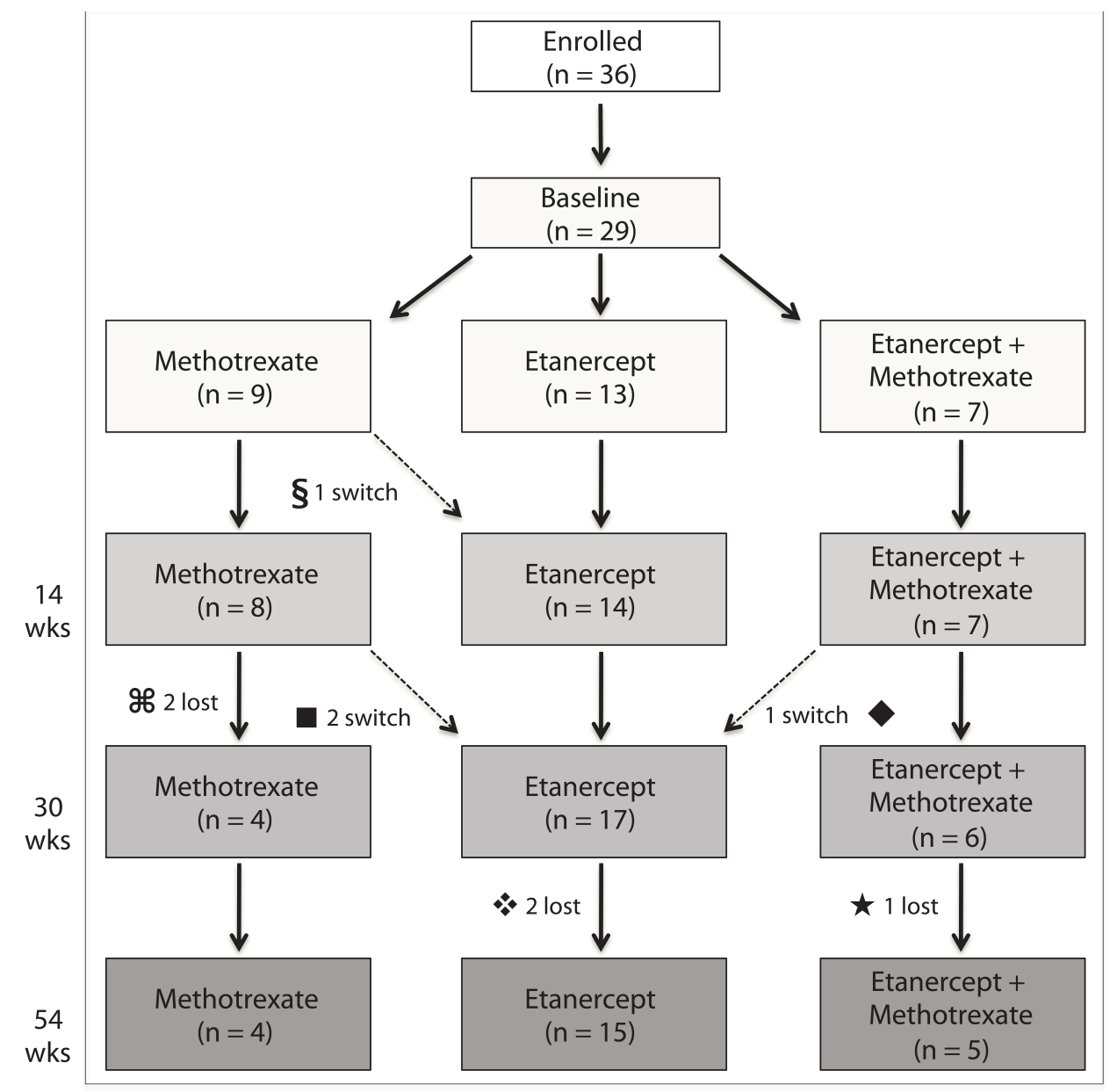

Figure 1. Flow chart of the study. \$ One patient switched to etanercept because of elevated serum transaminases. $\mathscr{H}$ One patient was lost to followup and 1 stopped methotrexate because of inefficacy. One patient switched to etanercept because of increase in liver enzymes and 1 because of nausea. $\bullet$ One patient stopped methotrexate as a result of rash and continued with etanercept. One patient stopped etanercept because of rash and 1 because of paresthesia. * One patient stopped methotrexate and etanercept owing to heart failure.

with etanercept alone, and 7 with the combined treatment MTX plus etanercept. At 6 weeks, 1 patient taking MTX switched to etanercept because of elevated serum transaminases. At 18 weeks, 1 patient taking MTX was lost to followup, 1 patient stopped MTX because of inefficacy and withdrew from the study, and 1 patient taking MTX switched to etanercept because of an increase of AST, ALT, and ALP levels. At 22 weeks, 1 patient on MTX switched to etanercept as a result of nausea, and 1 patient taking combination therapy stopped MTX because of rash and continued etanercept alone. At 30 weeks, 1 patient stopped MTX and etanercept owing to heart failure. At 38 weeks, 1 patient stopped etanercept because of rash, and at 46 weeks 1 patient stopped etanercept as a result of paresthesia.

Liver function and HCV activity. Liver function was assessed at entry, 14, 30, and 54 weeks of therapy. Comparisons were made between baseline and the other followup time points within each treatment group.
As shown in Figure 2, in patients taking etanercept alone there was no significant change in liver function between baseline and any followup timepoint in ALP $(90 \pm 13$ at entry, $105 \pm 13,105 \pm 19$, and $127 \pm 14$ at 14,30 , and 54 weeks, respectively), ALT ( $48 \pm 11$ at entry, $51 \pm 7,60 \pm 13$, and $47 \pm 10$ at 14,30 , and 54 weeks, respectively), AST (38 \pm 6 at entry, $39 \pm 7,46 \pm 10$, and $38 \pm 7$ at 14,30 , and 54 weeks, respectively) or GGT ( $43 \pm 8$ at entry, $50 \pm 13,55 \pm$ 23 , and $97 \pm 6$ at 14,30 , and 54 weeks, respectively). In patients taking MTX therapy, ALP levels were significantly increased from baseline $(97 \pm 12)$ to 14 weeks $(159 \pm 24$, $\mathrm{p}<0.05)$, then decreased at $30(125 \pm 33)$ and 54 weeks $(119$ $\pm 20)$ and were no longer significantly higher than baseline; other measures were not significantly elevated at any followup timepoint (ALT $35 \pm 7$ at entry, $42 \pm 14,43 \pm 20$, and $41 \pm 7$ at 14, 30, and 54 weeks, respectively; AST $36 \pm$ 2 at entry, $41 \pm 12,44 \pm 9$, and $38 \pm 7$ at 14,30 , and 54 weeks, respectively; GGT $29 \pm 5$ at entry, $21 \pm 2,35 \pm 8$, and 


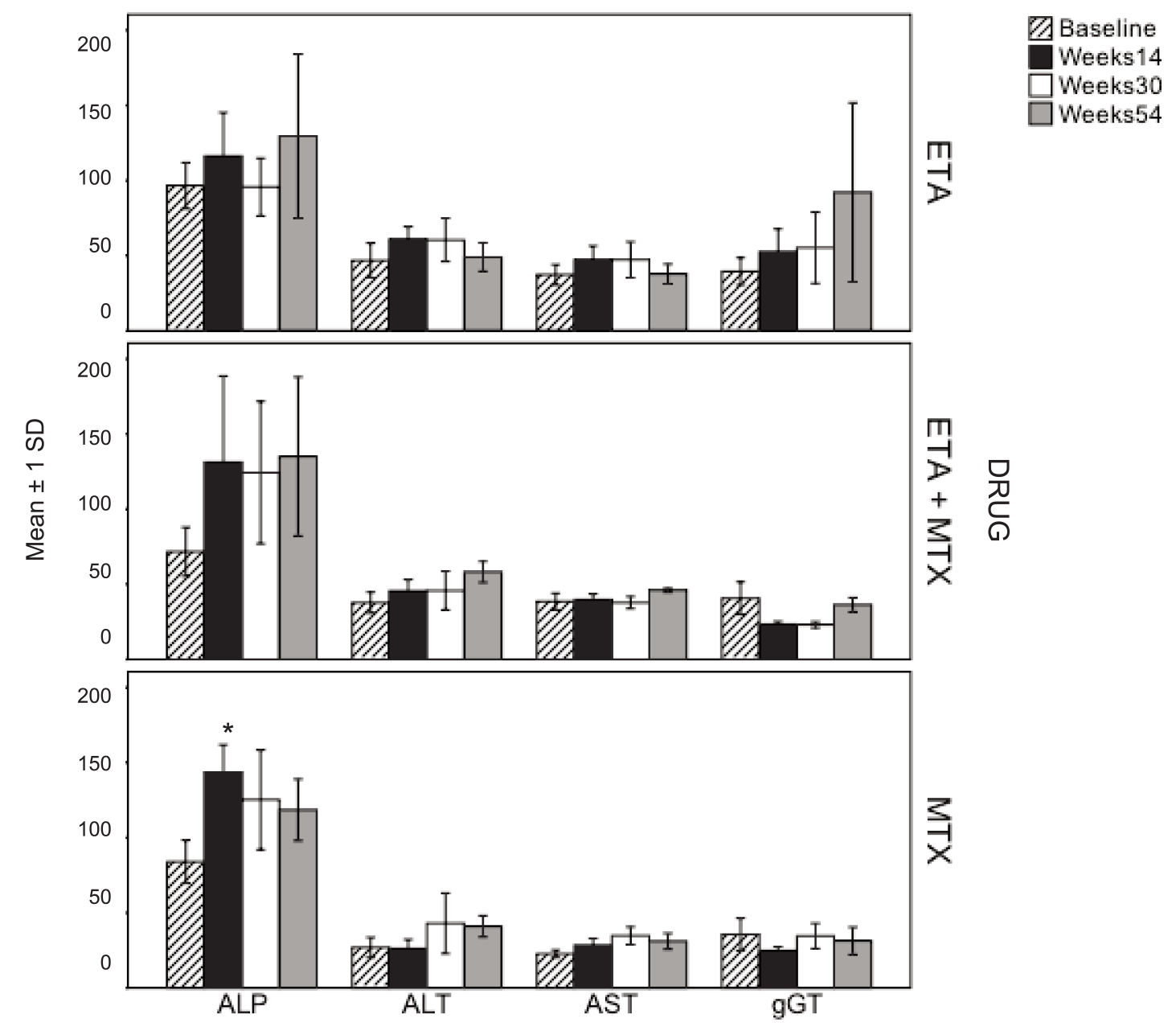

Figure 2. Trends in liver enzymes (mean $\pm 1 \mathrm{SD}$ ) in patients taking etanercept (ETA) alone, methotrexate (MTX) alone, and ETA + MTX at baseline and after 14, 30, and 54 weeks of therapy. $* \mathrm{p}<0.05$ vs baseline.

$30 \pm 6$ at 14, 30, and 54 weeks, respectively). In patients taking a combination of MTX and etanercept, the increases in ALP levels at the followup timepoints were not large enough to be significant $(87 \pm 9$ at entry, $146 \pm 32,115 \pm 34$, and $135 \pm 52$ at 14,30 , and 54 weeks, respectively), nor were there significant changes in other enzymes (ALT $44 \pm$
6 at entry, $44 \pm 5,42 \pm 10$, and $58 \pm 7$ at 14,30 , and 54 weeks; AST $39 \pm 7$ at entry, $38 \pm 5,33 \pm 10$, and $46 \pm 2$ at 14,30 , and 54 weeks; GGT $47 \pm 7$ at entry, $34 \pm 8,36 \pm 6$, and $36 \pm 4$ at 14, 30, and 54 weeks, respectively). In Table 2 , changes in albumin and total bilirubin levels, PT, and HCV viral load are summarized. No significant differences

Table 2. Serial measurements of albumin (ALB), bilirubin (BIL), prothrombin time (PT), and HCV viral load (expressed as log 10 of the detected values) in the 3 treatment groups at baseline, 14, 30, and 54 weeks. Values are shown as mean \pm 1 SD.

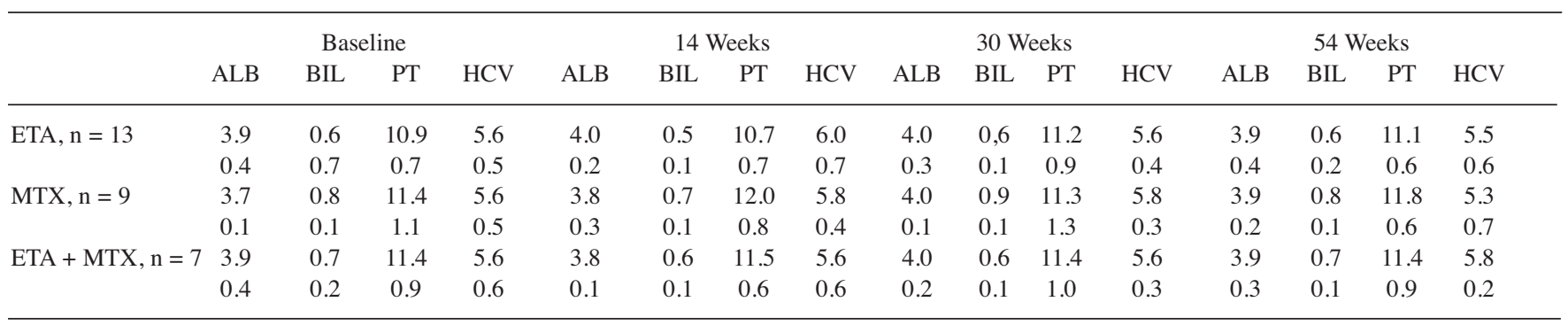

MTX: methotrexate; ETA: etanercept; HCV: hepatitis C virus. 
within the 3 arms of treatments were detected throughout the study. Serial liver US did not show significant changes during the study.

Safety and clinical response. Drugs were globally well-tolerated, with one single serious event, heart failure, occurring in the group taking etanercept in combination with MTX. With regard to mild side effects, in the MTX group 1 patient complained of nausea, in the MTX plus etanercept group a rash occurred in 1 patient, and in the etanercept group 1 patient complained of rash and another of paresthesia. The rate of therapy survival was $83 \%$ (24 out of 29 patients) at 54 weeks.

Clinical outcomes were assessed by measuring the DAS44 and functional disability (HAQ). DAS44 was $4.2 \pm$ 0.6 at entry, $3.1 \pm 1$ after 14 weeks, $2.6 \pm 1$ after 30 weeks, and $2.6 \pm 1$ after 54 weeks, the differences were statistically significant (Figure 3). The percentage of patients achieving a good EULAR response (a reduction of DAS44 $\geq 1.2$ ) was $44 \%$ at 14 weeks $(\mathrm{p}>0.05), 73 \%$ at 30 weeks $(\mathrm{p}<0.01)$, and $74 \%$ at 54 weeks $(\mathrm{p}<0.01)$. Also, the HAQ disability index showed a significant reduction, 1.25 (IQR 0.75) at baseline, 0.75 (IQR 0.81 ) at 14 weeks ( $\mathrm{p}=0.001$ vs baseline), 0.75
(IQR 1.0) at 30 weeks ( $\mathrm{p}=0.002$ vs baseline), and 0.63 (IQR 1.4) at 54 weeks ( $\mathrm{p}=0.04$ vs baseline).

\section{DISCUSSION}

In the past, the rheumatology community was afraid to use MTX in patients with RA and chronic HCV infection because of the intrinsic hepatotoxicity of $\mathrm{MTX}^{1}$ and because of the reduced capability of the immune system to downregulate the replication of $\mathrm{HCV}$. This concern has become more urgent since the entry of anti-TNF agents into the therapy of refractory RA, because anti-TNF drugs might have impaired the control of viral replication through the interferon pathway and fostered HCV-mediated liver damage.

In the early 1990s, serial liver biopsy was recommended to monitor possible development of cirrhosis in patients with RA taking MTX $^{10}$. Thereafter, this caveat has been downsized and it has been shown that longterm MTX treatment does not appear to be associated with the development of significant liver fibrosis ${ }^{11}$, and now, serum levels of transaminases are monitored to observe drug hepatotoxicity in clinical practice. Nevertheless, a multinational consensus of rheumatologists has developed evidence-based

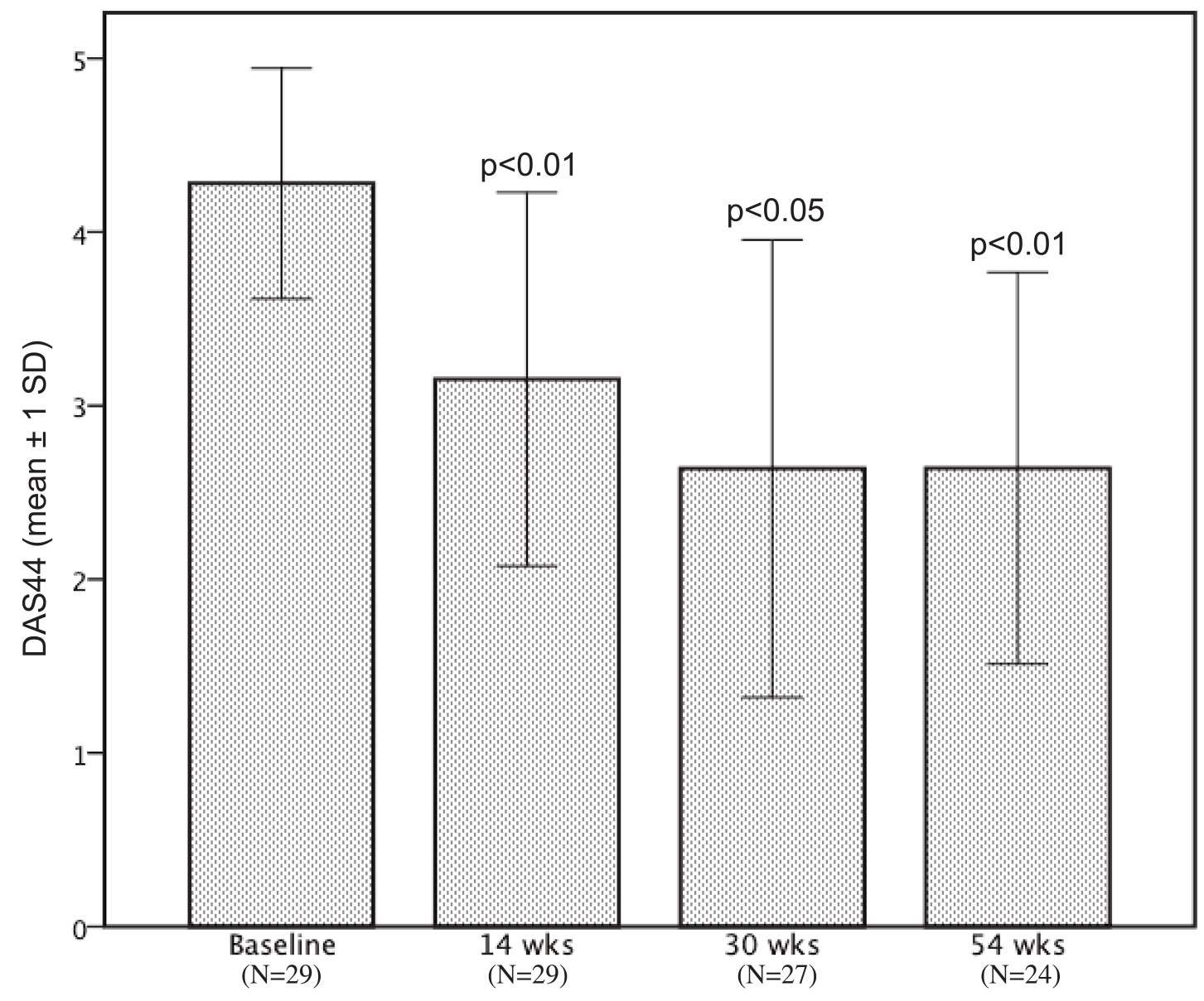

Figure 3. Disease activity score of 44 joints (DAS44, mean \pm 1 SD) at baseline and after 14,30 , and 54 weeks of therapy in overall patients. Statistical significance compared to baseline.

Personal non-commercial use only. The Journal of Rheumatology Copyright (c) 2014. All rights reserved. 
recommendations for the use of MTX in RA indicating $\mathrm{HCV}$ infection as an additional risk factor for MTX-induced hepatotoxicity ${ }^{12}$. But there is no recommendation for the treatment of patients with active RA and HCV infection, although in clinical practice low doses of glucocorticoids and disease-modifying antirheumatic drugs other than MTX and leflunomide (such as hydroxychloroquine or sulfasalazine) are currently used.

Some studies suggest that anti-TNF agents may play a direct role in HCV-mediated hepatic inflammation and fibrosis, and that they may not be detrimental to liver function in $\mathrm{HCV}$-infected patients. Levels of soluble anti-TNF receptors are significantly correlated with the severity of HCV-related liver disease ${ }^{13,14}$, and TNF- $\alpha$ activation pathways are directly involved in liver inflammation $^{15}$ and hepatic steatosis ${ }^{16}$ in patients with chronic HCV infection. Further, TNF- $\alpha$ inhibition has been shown to have an antiviral effect by promoting hepatocyte apoptosis and preventing hepatocyte regeneration in a transgenic mouse model of chronic hepatitis $\mathrm{C}^{17}$. On this basis, etanercept has been used as adjuvant therapy to interferon and ribavirin in patients with $\mathrm{HCV}$ chronic infection and it has been shown that viral activity was significantly lower in patients taking etanercept than in those who did not after 24 weeks of treatment ${ }^{18}$.

These data represented the rationale that anti-TNF drugs were safer than MTX in patients with RA and chronic HCV infection. Peterson, et al have retrospectively studied 16 patients with RA and HCV infection treated with etanercept or infliximab, 4 of whom were taking combination therapy with MTX $^{8}$. They also prospectively enrolled 8 patients with RA who were treated with etanercept alone and prednisolone. Anti-TNF drugs were globally well tolerated without significant increases of liver enzymes or HCV viral load ${ }^{8}$. In another retrospective study, infliximab and etanercept did not worsen liver function in 5 patients with active RA and HCV infection ${ }^{7}$. Two further retrospective analyses have confirmed that therapy with TNF- $\alpha$ inhibitors (adalimumab, infliximab, etanercept) is safe in this setting for patients $5,6,7,8,9$. The major weaknesses of these studies were the retrospective design and the lack of assessment of the association of anti-TNF drugs with MTX, which is the anchor drug for almost all therapies with biologics. These publications have been thoroughly reviewed by Brunasso, et $a l^{19}$, who reported 91 patients with RA and HCV infection globally treated with anti-TNF drugs and stated that there is a lack of prospective controlled clinical trials.

In our study, we focused on the safety and efficacy of the combination of MTX and etanercept in patients with RA and chronic HCV infection. For ethical reasons, we recruited patients with RA and mild hepatitis, while those with severe hepatitis or cirrhosis were excluded. Twenty-nine patients were randomly assigned to receive MTX or etanercept alone, or the 2 drugs together, and prospectively monitored over 54 weeks. The therapy was well tolerated, and 24 out of 29 patients $(83 \%)$ were still under treatment after 54 weeks. Serum levels of AST, ALT, ALP, GGT, albumin, bilirubin, and PT did not significantly change in all 3 groups of therapy, nor did HCV viral load. Only in the MTX arm did we detect a significant increase of ALP levels at 14 weeks that faded afterward. Our findings are in agreement with literature reporting a low global rate $(8.8 \%)$ of patients with RA who had elevated liver enzymes or worsening of HCV viral load while taking anti-TNF drugs ${ }^{19}$. Of note, to our knowledge, this is the largest cohort reported in the literature of patients with RA and $\mathrm{HCV}$ infection $(n=16)$ taking MTX, alone or in combination, and only 1 patient dropped out owing to elevated serum transaminases, suggesting that $\mathrm{HCV}$ infection might not increase the risk of liver toxicity by MTX. We also observed a good clinical response because the DAS44 and HAQ scores significantly decreased at 14 weeks with a further reduction up to 54 weeks. No significant differences of DAS44 and HAQ were detected among the 3 treatments at any timepoint (data not shown).

However, some weaknesses of our study must be highlighted. Although it was the largest cohort in this setting, the sample size was relatively small and reduces the statistical power of our study. However, the results on safety are consistent with those extrapolated from various studies on 91 patients with RA and HCV infection treated with different anti-TNF agents globally ${ }^{19}$. Despite being prospective and randomized, our trial had an open-label design and only patients with RA and mild HCV related hepatitis were enrolled. Notwithstanding, we provided evidence that a definite subset of patients with RA and chronic HCV infection and compensated liver function may be successfully treated using etanercept and MTX alone or in combination without increasing the risk of hepatotoxicity and HCV replication. Instead, data on the outcomes of anti-TNF therapy in patients with RA and HCV infection and advanced fibrosis are still lacking.

\section{REFERENCES}

1. Zachariae H, Søgaard H, Heickendorff L. Methotrexate-induced liver cirrhosis. Clinical, histological and serological studies - a further 10-year follow-up. Dermatology 1996;192:343-6.

2. Vassilopoulos D, Calabrese LH. Management of rheumatic disease with comorbid HBV or HCV infection. Nat Rev Rheumatol 2012;8:348-57.

3. Bellisai F, Giannitti C, Donvito A, Galeazzi M. Combination therapy with cyclosporine A and anti-TNF-alpha agents in the treatment of rheumatoid arthritis and concomitant hepatitis $\mathrm{C}$ virus infection. Clin Rheumatol 2007;26:1127-9.

4. Cavazzana I, Ceribelli A, Cattaneo R, Franceschini F. Treatment with etanercept in six patients with chronic hepatitis $\mathrm{C}$ infection and systemic autoimmune diseases. Autoimmun Rev 2008;8:104-6.

5. Ferri C, Ferraccioli G, Ferrari D, Galeazzi M, Lapadula G, Montecucco C, et al. Safety of anti-tumor necrosis factor-alpha therapy in patients with rheumatoid arthritis and chronic hepatitis C virus infection. J Rheumatol 2008;35:1944-9.

6. Li S, Kaur PP, Chan V, Berney S. Use of tumor necrosis

Personal non-commercial use only. The Journal of Rheumatology Copyright ()$^{2014}$. All rights reserved. 
factor-alpha (TNF-alpha) antagonists infliximab, etanercept, and adalimumab in patients with concurrent rheumatoid arthritis and hepatitis B or hepatitis $\mathrm{C}$ : a retrospective record review of 11 patients. Clin Rheumatol 2009;28:787-91

7. Parke FA, Reveille JD. Anti-tumor necrosis factor agents for rheumatoid arthritis in the setting of chronic hepatitis $\mathrm{C}$ infection. Arthritis Rheum 2004;51:800-4.

8. Peterson JR, Hsu FC, Simkin PA, Wener MH. Effect of tumour necrosis factor alpha antagonists on serum transaminases and viraemia in patients with rheumatoid arthritis and chronic hepatitis C infection. Ann Rheum Dis 2003;62:1078-82.

9. Uda H, Kuhara M, Nishimoto N, Saiki O. Progression of viraemia during treatment with infliximab in a patient with rheumatoid arthritis and chronic hepatitis C infection. BMJ Case Rep 2009;2009.

10. Erickson AR, Reddy V, Vogelgesang SA, West SG. Usefulness of the American College of Rheumatology recommendations for liver biopsy in methotrexate-treated rheumatoid arthritis patients. Arthritis Rheum 1995;38:1115-9.

11. Hashkes PJ, Balistreri WF, Bove KE, Ballard ET, Passo MH. The long-term effect of methotrexate therapy on the liver in patients with juvenile rheumatoid arthritis. Arthritis Rheum 1997; 40:2226-34.

12. Visser K, Katchamart W, Loza E, Martinez-Lopez JA, Salliot C, Trudeau J, et al. Multinational evidence-based recommendations for the use of methotrexate in rheumatic disorders with a focus on rheumatoid arthritis: integrating systematic literature research and expert opinion of a broad international panel of rheumatologists in the 3E Initiative. Ann Rheum Dis 2009;68:1086-93.
13. Macías J, Sánchez-Quijano A, Pineda JA, Abad MA, Rubio A, Rosa $\mathrm{R}$, et al. Minimal liver injury in chronic hepatitis $\mathrm{C}$ virus infection is associated with low levels of soluble TNF-alpha/Fas receptors and acquisition in childhood. Liver 2001;21:410-4.

14. Zylberberg H, Rimaniol AC, Pol S, Masson A, De Groote D, Berthelot $\mathrm{P}$, et al. Soluble tumor necrosis factor receptors in chronic hepatitis $\mathrm{C}$ : a correlation with histological fibrosis and activity. J Hepatol 1999;30:185-91.

15. Kinnman N, Andersson U, Hultcrantz R. In situ expression of transforming growth factor-beta1-3, latent transforming growth factor-beta binding protein and tumor necrosis factor-alpha in liver tissue from patients with chronic hepatitis C. Scand J Gastroenterol 2000;35:1294-300

16. Mundt B, Wirth T, Zender L, Waltemathe M, Trautwein C, Manns MP, et al. Tumour necrosis factor related apoptosis inducing ligand (TRAIL) induces hepatic steatosis in viral hepatitis and after alcohol intake. Gut 2005;54:1590-6.

17. Brenndörfer ED, Weiland M, Frelin L, Derk E, Ahlén G, Jiao J, et al. Anti-tumor necrosis factor $\alpha$ treatment promotes apoptosis and prevents liver regeneration in a transgenic mouse model of chronic hepatitis C. Hepatology 2010;52:1553-63.

18. Zein NN, Etanercept Study Group. Etanercept as an adjuvant to interferon and ribavirin in treatment-naive patients with chronic hepatitis $\mathrm{C}$ virus infection: a phase 2 randomized, double-blind, placebo-controlled study. J Hepatol 2005;42:315-22.

19. Brunasso AM, Puntoni M, Gulia A, Massone C. Safety of anti-tumour necrosis factor agents in patients with chronic hepatitis C infection: a systematic review. Rheumatology 2011;50:1700-11. 